\title{
Aspect Ratio Dependence of Isotropic-Nematic Phase Separation of Nanoplates in Gravity
}

\author{
Abhijeet Shinde ${ }^{1}$, Xuezhen Wang ${ }^{1,2}$, Yi-Hsien $\mathrm{Yu}^{3}$, and Zhengdong Cheng ${ }^{1,2,3,4}$ \\ ${ }^{1}$ Artie McFerrin Department of Chemical Engineering, Texas A\&M University, College Station, TX; ${ }^{2}$ Mary Kay \\ O’Conner Process Safety Center, Artie McFerrin Department of Chemical Engineering, Texas A\&M University, \\ College Station, TX; ${ }^{3}$ Department of Material Science and Engineering, Texas A\&M University, TX; ${ }^{4}$ Professional \\ Program in Biotechnology, Texas A\&M University, College Station, TX
}

\begin{abstract}
We studied isotropic-nematic (I-N) phase separation via gravity sedimentation in suspensions of plate-like colloidal particles of identical thickness but different lateral sizes (diameters). It is well-known that I-N phase transition occurs at a higher concentration for particles with larger aspect ratio (thickness/diameter) than for particles with smaller aspect ratio. Here we report that for the larger aspect ratios of nanoplates, gravity-driven I-N phase separation is faster. In a homogenously mixed I-N biphasic suspension of nanoplates, nematic tactoids nucleate, grow, and then undergo sedimentation in gravity, leading to the formation of a clear horizontal interface between the I and $\mathrm{N}$ phase. For I-N coexistent suspension of nanoplates with different aspect ratios but the same amount of nematic fractions, the larger the aspect ratio, the faster the formation of nematic
\end{abstract}

Key words: Liquid Crystal; Nanoplates; Isotropic; Nematic; Colloidal Disks; Phase Separation; Kinetics; Tactoids; Aspect Ratio; Lyotropic

Correspondence to: Zhengdong Cheng 220 Jack E Brown Engineering Building 3122 TAMU

College Station, TX 77843

Telephone: 979-845-3413

E-mail: zcheng@tamu.edu tactoids and interface between isotropic liquid and nematic liquid crystal phase. The tactoid formation rate is governed by the rotational and translational diffusion rates, which are faster at larger aspect ratios. The time required for I-N separation ( $\mathrm{t}^{*}$, seconds) varies inversely with the mean aspect ratio $(<\xi>)$ of nanoplates and follows the relation, $t^{*}=\alpha\langle\xi\rangle^{n}$, where $\alpha=0.97 \pm$ $1.30 \mathrm{~s}$ and $n=-2.1 \pm 0.2$. The phase separation kinetics studied in our experiments offers guidance for the selection of aspect ratio of nanoplates for samples to be studied at the International Space Station (ISS).

\section{INTRODUCTION}

Colloidal crystallization of hard spheres is well-studied in microgravity (Zhu et al., 1997; Cheng et al., 1999; Cheng et al., 2002). However, there is a need for similar studies of lyotropic liquid crystalline phase transitions and phase transition kinetics in suspensions of twodimensional colloids, also known as nanoplates, which are building blocks of naturally abundant clay. Liquid crystals are matter in a state that shows symmetry and other properties intermediate between those of liquids and solids. For example, they can flow like liquids and have long range spatial or orientational order like solids, but only in one or two dimensions. Nematic liquid crystal has a long-range orientational order of constituent mesogens (molecules or particles), which means 
that the constituent molecules or nanoparticles are aligned along a certain direction called "nematic director." Nanoplate suspensions show novel liquid crystalline nematic (van der Kooij et al., 2000), smectic (Sun et al., 2009; Kleshchanok et al., 2012), and columnar (van der Kooij et al., 2000) phases. The rich liquid crystalline phase behavior of nanoplates makes it even more interesting to study their phase transition kinetics in microgravity. In preparation for conducting nanoplate liquid crystal formation study in the microgravity environment at the International Space Station (ISS), we studied the nanoplate size dependence of isotropic (I) liquid and nematic liquid crystal $(\mathrm{N})$ phase separation kinetics under the influence of gravity. The Onsager theory (Onsager, 1949) for anisotropic colloidal particles predicts that the lesser the aspect ratio (thickness/diameter) of the particles, the lesser the concentration required to form a nematic phasethis has been verified experimentally (Mejia et al., 2012). Very low aspect ratio nanoplates have recently been found to exhibit richer phase behavior, including chiral phases (Xu and Gao, 2011). However, the kinetics of liquid crystalline phase formation can be very slow for nanoplates with very low aspect ratio. Thus an optimum value of size needs to be chosen in order to ensure successful completion of desired phase transition studies in the limited flight time on ISS. Hence, we tried to study how aspect ratio affects the kinetics of the nematic phase formation from metastable fluid state. Small nematic domains that nucleate from metastable fluids are called tactoids. In the microgravity environment the problem of sedimentation of nematic tactoids can easily be avoided, and the growth of tactoidstheir coarsening-can be studied in detail. Different shapes of tactoids and their director field distribution have been reported previously (Verhoeff et al., 2011); however, little has been reported on the timeframe for the I-N separation and its dependence on size (diameter) or thickness.

\section{MATERIALS AND METHODS}

Nanoplate suspensions used in this study were obtained by using a two-step process. First, pristine $\alpha$-zirconium phosphate disks of
$\mathrm{ZrH}\left(\mathrm{PO}_{4}\right) \cdot \mathrm{H}_{2} \mathrm{O} \quad$ (abbreviated as $\alpha-\mathrm{ZrP}$, henceforth), were obtained using either a hydrothermal (Shuai et al., 2013) or reflux (Clearfield and Stynes, 1964) method. Second, pristine $\alpha$-ZrP was exfoliated into monolayer $\mathrm{ZrP}$ platelets (henceforth called nanoplates) using tetra-(n) butyl ammonium hydroxide, as described in Kim et al. (1997). Monolayer ZrP has a single plane of $\mathrm{Zr}$ atoms with $\mathrm{P}, \mathrm{O}$, and $\mathrm{H}$ atoms on both sides of the $\mathrm{Zr}$ atomic plane. The details of bonding in monolayer can be found in Kim et al. (1997). Aqueous suspensions of nanoplates with five different mean diameters were obtained and categorized into five groups-group 1 had the lowest size (diameter) nanoplates and group 5 had the highest size nanoplates. Pristine $\alpha-\mathrm{ZrP}$ disks used to obtain nanoplates suspensions of group 2 to group 5 were obtained using the hydrothermal method, whereas that used for group 1 suspensions were obtained using the reflux method. The advantage of the hydrothermal method is that one can make disks of mean size ranging from $500 \mathrm{~nm}$ to $1500 \mathrm{~nm}$ by controlling the reaction conditions (Shuai et al., 2013), and also can keep polydispersity of the pristine $\alpha$-ZrP disks relatively small ( $<30 \%)$.

Figure 1 shows an electron micrograph of pristine $\alpha$-ZrP (used in sample group 5). We made use of Dynamic Light Scattering (DLS) to measure the diameter $(d)$ of monolayer $\mathrm{ZrP}$ nanoplates obtained in the second step (He et al., 2010). DLS gives the hydrodynamic size from which one may calculate the lateral size. Making use of the already established fact that monolayers have a uniform thickness $(l)$ of $2.68 \mathrm{~nm}$ (Sun et al., 2009), the lateral size (diameter) distributions of nanoplates in five different groups of suspensions were obtained, as shown in Figure 2.

Five different mother suspensions with different lateral sizes of monolayer $\mathrm{ZrP}$ were prepared and each of them was diluted with milliQ water to obtain different volume fractions of nanoplates, which exhibit isotropic, I-N coexistence, and full-nematic phases. The suspensions were placed in $1 \mathrm{~mL}$ cylindrical vials, homogenized by hand shaking, and then placed between a pair of crossed-polarizers in increasing order of concentration. Their snapshots were taken as a function of time. 


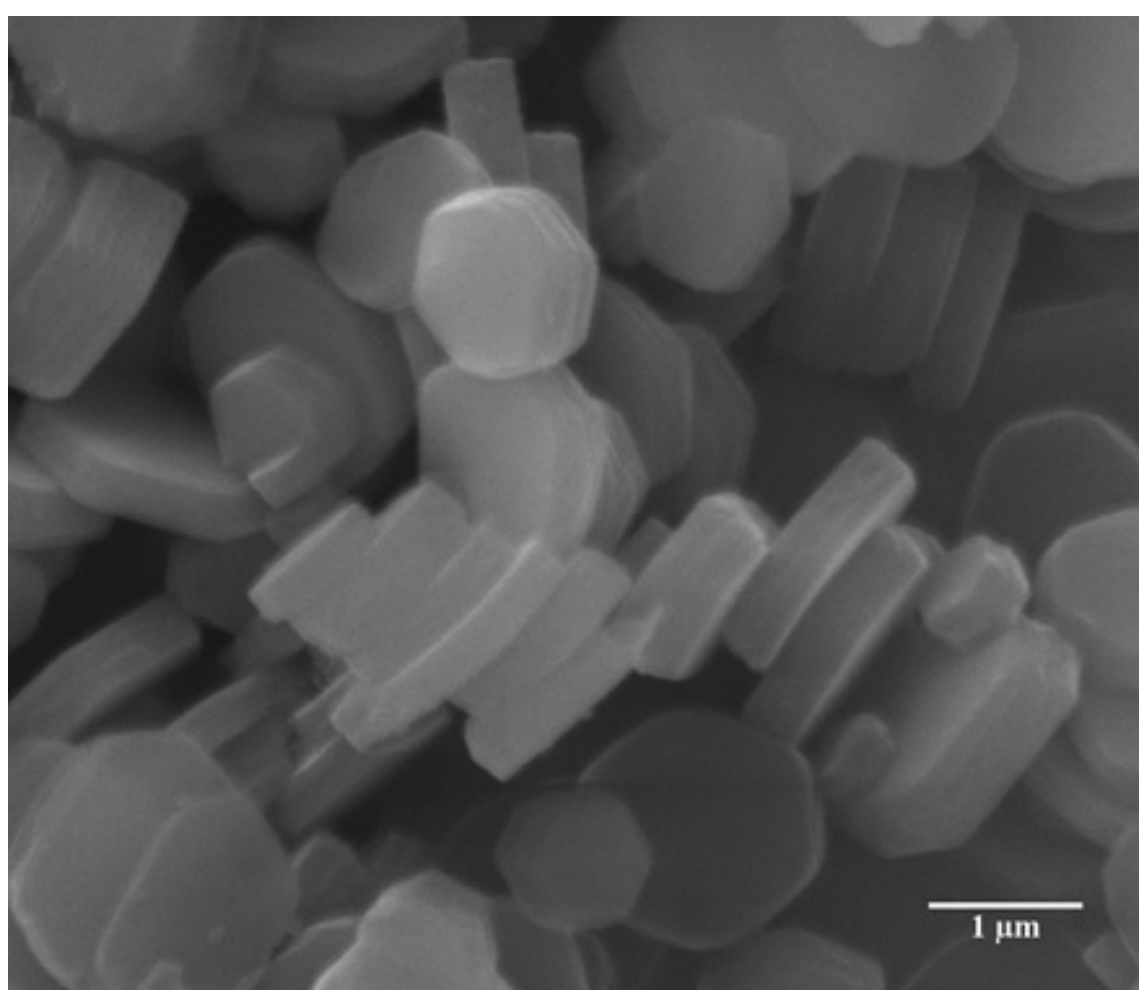

Figure 1. A scanning electron microscope (SEM) image of the pristine ZrP disks.

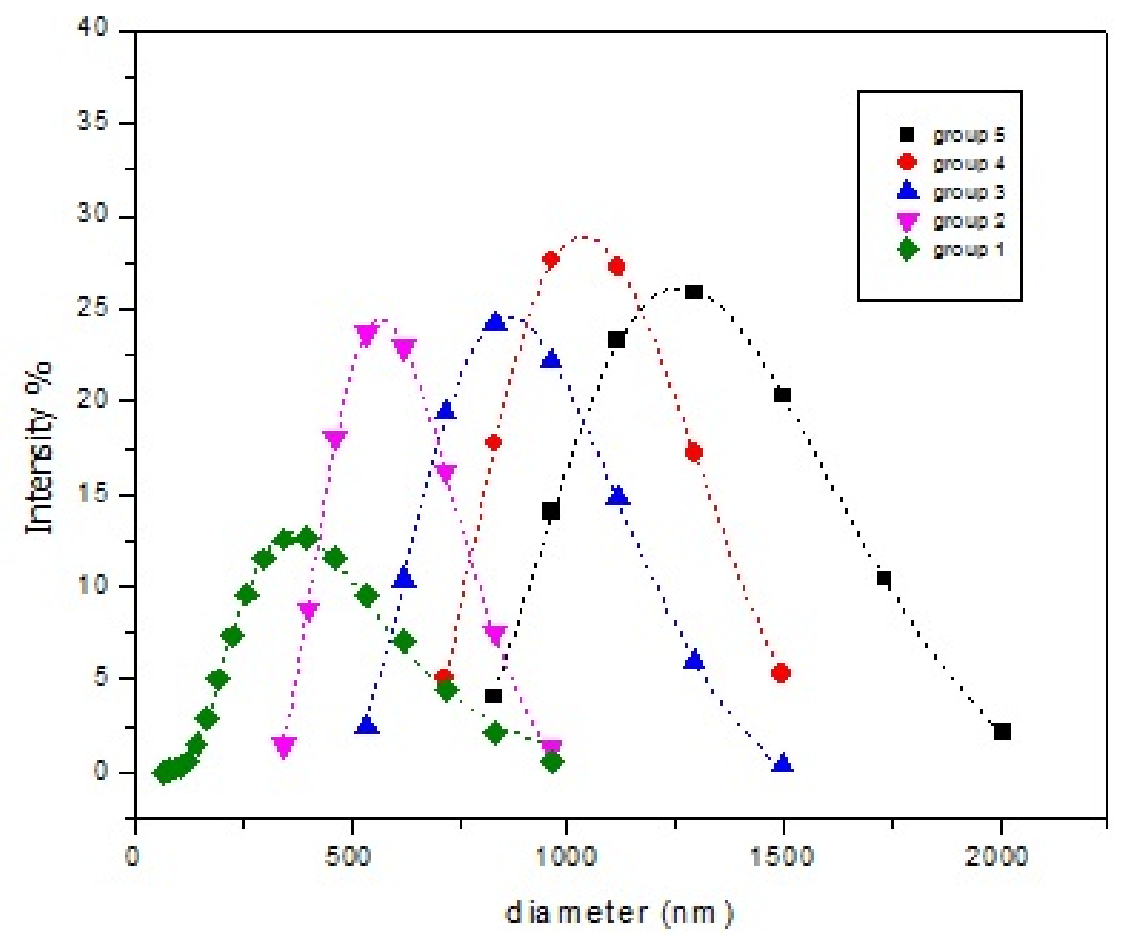

Figure 2. Lateral size (diameter) distributions of nanoplates in group 1 to group 5 suspensions used in this study. The values were measured using Dynamic Light Scattering (DLS) and they were fit to the log-normal distribution function. 


\section{RESULTS}

Figure 3 shows snapshots of the group 1 suspension, which had an average nanoplate size (diameter) of $454 \mathrm{~nm}$ and a nanoplate volume fraction, $\phi=2.8 \%$. Initially (i.e., $\mathrm{t}=0$ ), the suspension was homogenized by gentle hand shaking and turning the vial upside down while making sure that no air bubble is trapped inside the susspension. In a matter of seconds, the sample nucleated nematic liquid crystalline droplets, also known as tactoids (Verhoeff et al., 2011), grew larger with time and underwent sedimentation, due to gravity. After the nematic tactoids settled to the bottom of the vertical tube, a clear interface between the isotropic and nematic phase was established. The time elapsed between homogenization of the sample and the establishment of a clear interface between I and $\mathrm{N}$ phase, is defined as the time required for I-N separation $\left(\mathrm{t}^{*}\right)$. At this time, the gravitational sedimentation of tactoids was judged to have finished. We monitored kinetics of I-N separation in group 1 to group 5 suspensions. Mean aspect ratios (defined as ratio of thickness to diameter) of nanoplates in different groups are listed in Table 1.

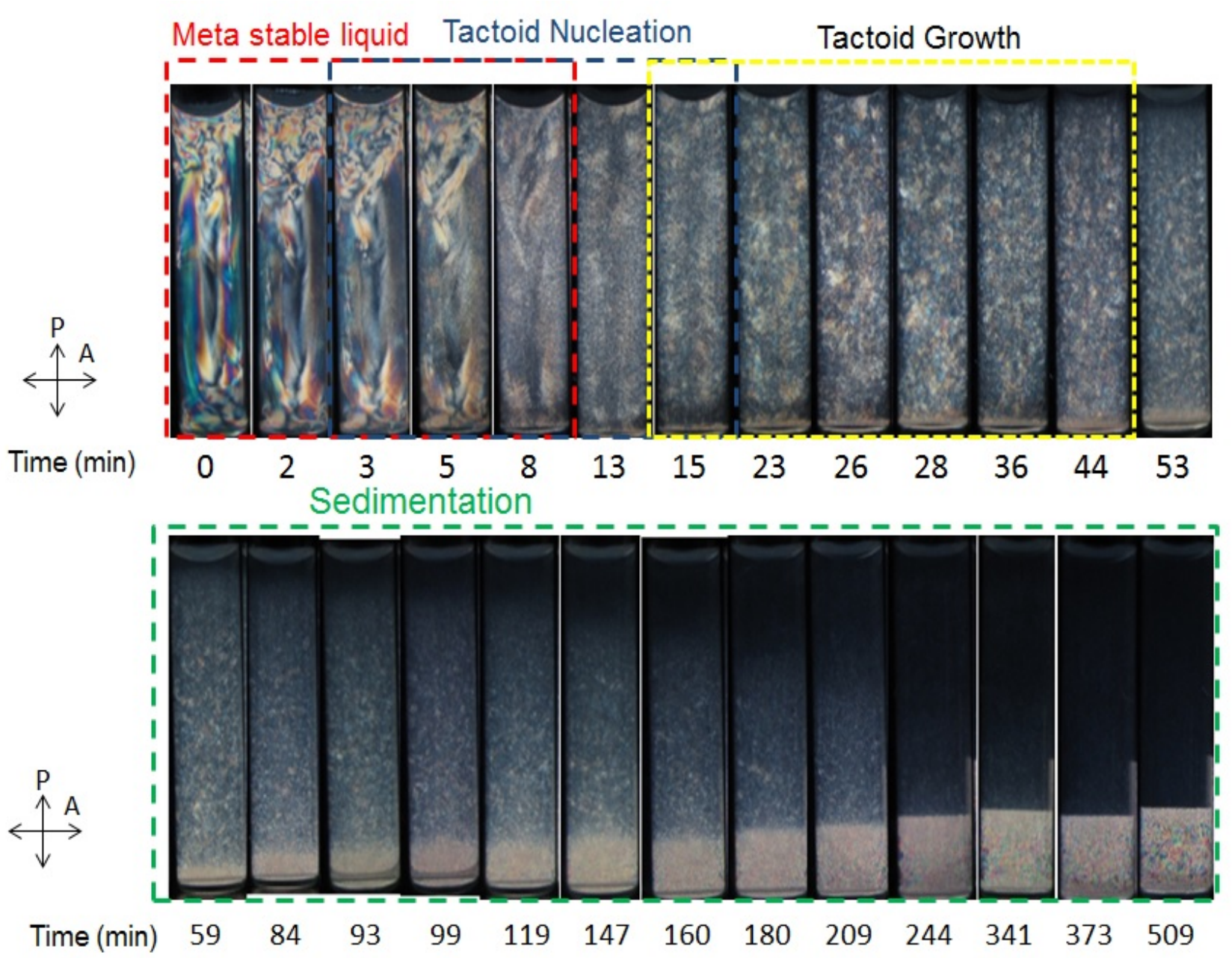

Figure 3. Snapshots of a group 1 nanoplate suspension $\left(\xi=0.0059_{-0.0018}^{+0.0049}\right)$ between crossed polarizers with a ZrP platelet volume fraction, $\phi=1.75 \%$, taken as a function of time. In the beginning, the suspension was in a metastable liquid state and then underwent the nucleation and growth of tactoids, followed by tactoid sedimentation, which was completed at time, $t^{*}$, when a clear interface between isotropic and nematic phases had established. 
Table 1. Lateral diameters of nanoplates in five different types of suspensions used in this study.

\begin{tabular}{|c|c|c|c|c|}
\hline Group & $\begin{array}{c}\text { Average Diameter } \\
(<\mathrm{d}>) \text { in } \mathrm{nm}\end{array}$ & $\begin{array}{c}\text { Standard Deviation }(\sigma) \text { in } \\
\mathrm{nm}\end{array}$ & $\begin{array}{c}\text { Polydispersity } \\
(\Delta=\sigma /<\mathrm{d}>)\end{array}$ & $\begin{array}{c}\text { Aspect Ratio } \\
(\xi=l / \mathrm{d})\end{array}$ \\
\hline 1 & 454 & 208 & $46 \%$ & $0.0059_{-0.0018}^{+0.0049}$ \\
\hline 2 & 616 & 137 & $28 \%$ & $0.0045_{-0.0008}^{+0.0012}$ \\
\hline 3 & 933 & 212 & $28 \%$ & $0.0028_{-0.0005}^{+0.0008}$ \\
\hline 4 & 1105 & 216 & $25 \%$ & $0.0025_{-0.0004}^{+0.0006}$ \\
\hline 5 & 1356 & 288 & $26 \%$ & $0.0020_{-0.0003}^{+0.0005}$ \\
\hline
\end{tabular}

The value of I-N separation time was quantitatively obtained by image analysis. Grayscale values of all pixels in the image were obtained using IDL 6.2 image analysis software. The ratio of intensity (I) of the light transmitted through sample and crossed polarizers to the intensity $\left(\mathrm{I}_{0}\right)$ of light input to the polarizer was measured by standard charge-coupled device (CCD) relation, $\frac{I}{I_{0}}=\left(\frac{\text { GrayscaleValue }}{255}\right)^{2.4}$. The transmittance values along a vertical line at the center of vials shown in Figure 3 were analyzed from bottom to the top as a function of time, as shown in Figure 4(a). A sharp decrease in transmittance profile corresponds to the interface between isotropic and nematic phase, as annotated in Figure 4(a). The area under this transmission curve for values of height above the I-N interface height represents the amount of unsettled tactoids, which decreases first (trend 1) and remains relatively constant (trend 2) at a value close to zero. The value of $t^{*}$ is determined by the intersection of the two trends and is demonstrated in Figure 4(b).

Figure 5 demonstrates the I-N phase separation process for various concentrations of nanoplates. All the samples in Figure 5(b) correspond to the decreasing trend in Figure 4(a), whereas all samples in Figure 5(c) correspond to trend 2 of Figure 4(b); t* corresponds to a time in between 5(b) and 5(c).

Similar analyses were done for suspensions of other groups having different aspect ratios of nanoplates. Crossed polarizer snapshots, taken at t $=0$ and at $t$ close to $t^{*}$, are shown in Figure 6 . The nanoplate volume fractions are labeled at the bottom of each sample. It can be seen that the suspensions with an average platelet size of 454 nm (group 1) took less than 10 hours to complete I-N separation. As the platelet size increases, the required time for I-N separation also increases; and for suspensions with an average platelet size of $1356 \mathrm{~nm}$ (group 5), it took about 100 hours. The dependence of $t^{*}$ on aspect ratio is plotted in Figure 7. As predicted by the Onsager theory (Onsager, 1949) and verified with previous experiments (Mejia et al., 2012), the suspensions with large aspect ratio require a higher concentration of platelets to realize the nematic phase; this was confimed by Figure 6. For the $50 \%$ nematic phase, the volume fractions were $2.1 \%, 1.03 \%, 0.7 \%, 0.55 \%$, and $0.45 \%$ for suspensions with average platelet sizes (diameters) of $454 \mathrm{~nm}(<\xi>=0.0059), 616 \mathrm{~nm}$ ( $<\xi>=0.0045), 933 \mathrm{~nm}(<\xi>=0.0029), 1105$ $\mathrm{nm}(<\xi>=0.0025)$, and $1356 \mathrm{~nm}(<\xi>=$ $0.0020)$, respectively.

\section{DISCUSSION}

Figure 7 shows that the time required for I-N separation $\left(t^{*}\right)$ varies inversely with the mean aspect ratio of nanoplates and follows a power law relation: $t^{*}=\alpha\langle\xi\rangle^{n}$, where $\alpha=0.97 \pm$ $1.30 \mathrm{~s}$ and $n=-2.1 \pm 0.2$. The $\mathrm{I}-\mathrm{N}$ phase separation time is affected by nucleation rate of tactoids, as well as by the sedimentation speed of 


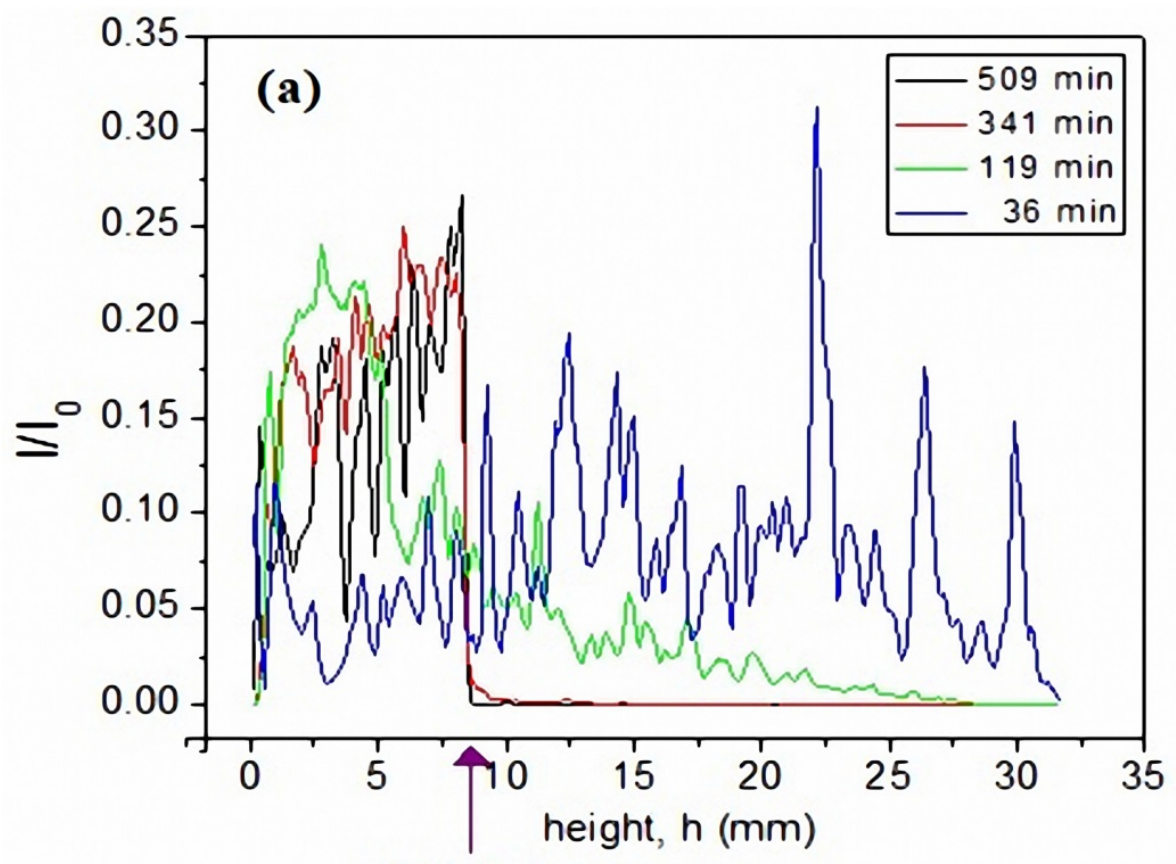

I-N interface

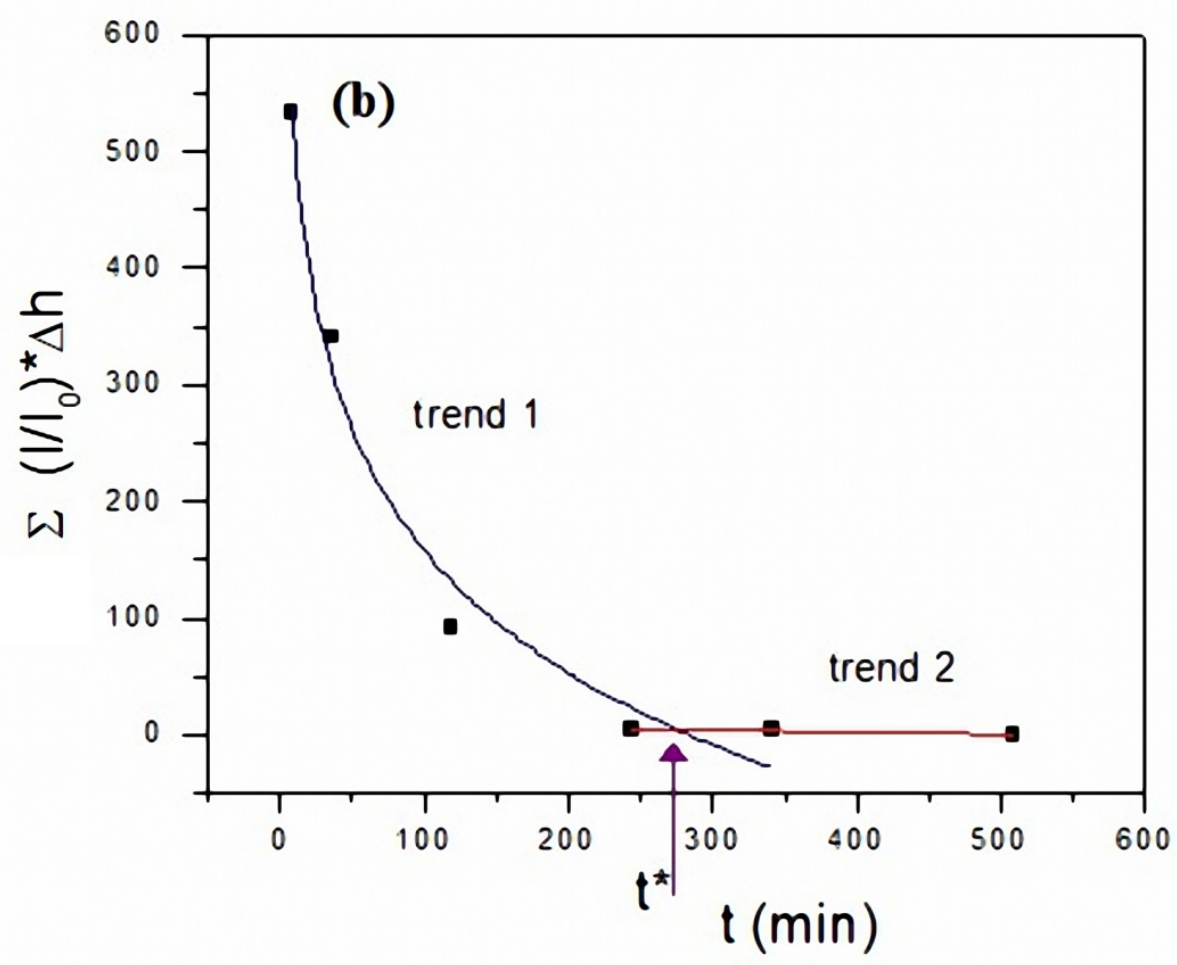

Figure 4. (a) Transmittance profiles along vertical line in group 1 suspension with $I / I_{0}$ plotted at different times. Position of $\mathrm{I}-\mathrm{N}$ interface is pointed with an arrow. The transmittance profile shows a sudden jump at 341 min, indicating the establishment of I-N interface. (b) Area under the transmittance curve in (a) for the region above the $\mathrm{I}-\mathrm{N}$ interface was plotted as a function of time. Time $\mathrm{t}^{*}$ corresponds to the point where area becomes zero. 

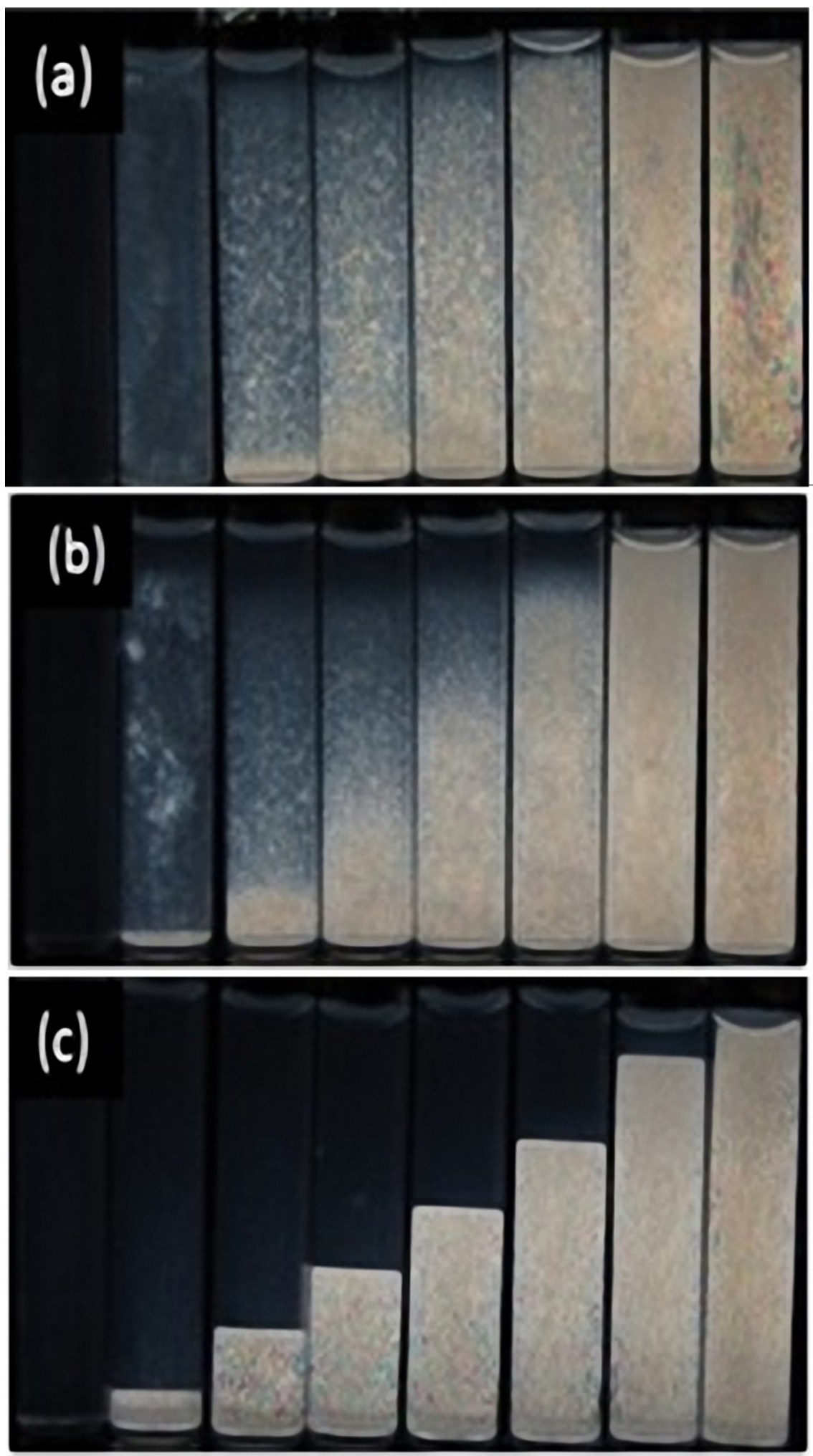

Figure 5. Crossed polarizers images of group 1 nanoplate suspensions at various concentrations taken at (a) 1 h (b) $2 \mathrm{~h}$ (c) $5.5 \mathrm{~h}$ after homogenization. Left to right, $\phi=0.0140,0.0158,0.0175,0.0193,0.0210,0.0228$, 0.0280, and 0.0350 . 


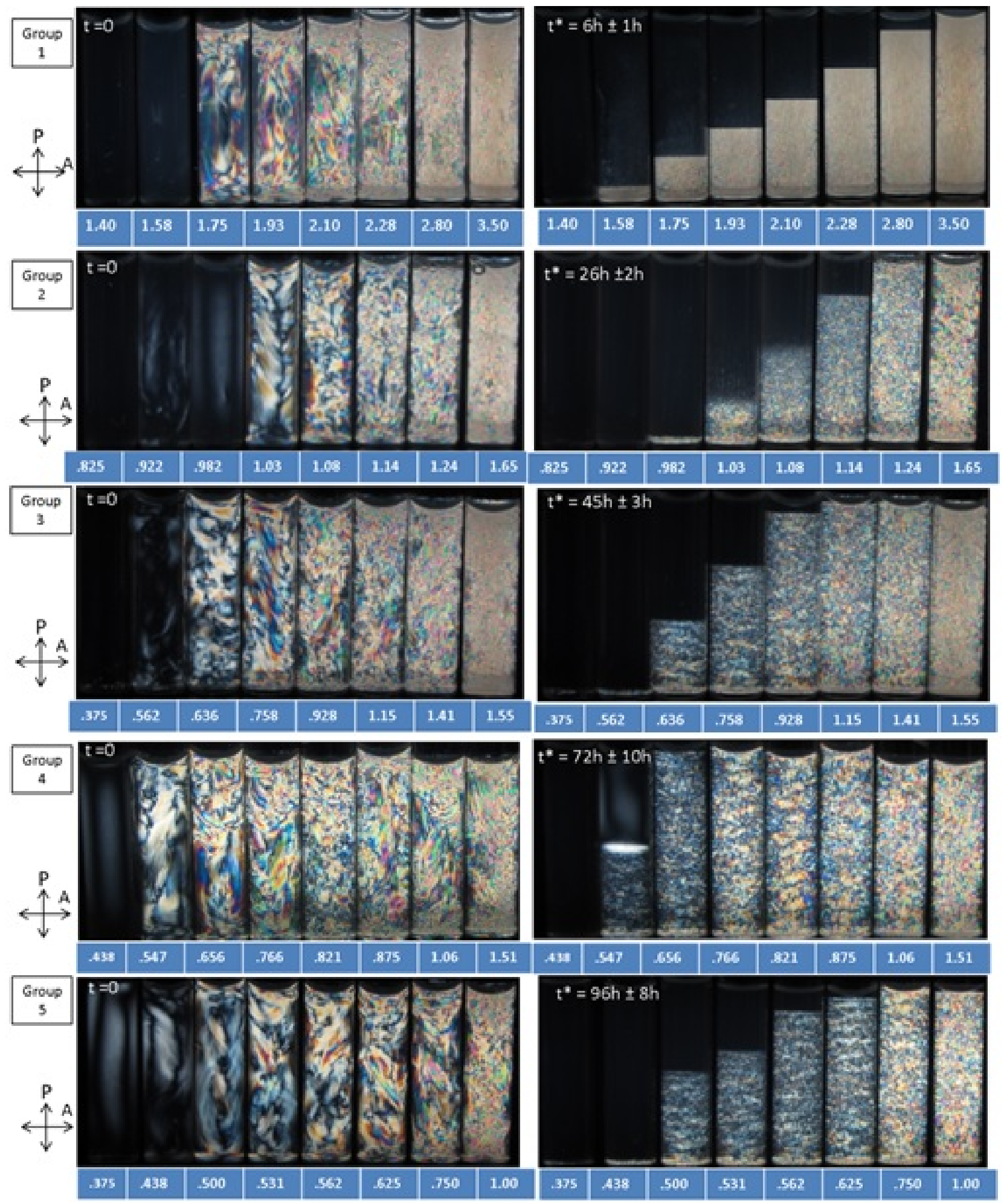

Figure 6. Crossed polarizer images of aqueous suspensions of different aspect ratio and different concentrations of $\alpha$-ZrP nanoplates. Left column shows textures of suspensions immediately after homogenization and the right column shows images when a clear interface between the Isotropic (I) and Nematic (N) phase was established. The ZrP nanoplate percentage by volume in each vial is labeled below. Group numbers of different samples are indicated on the left side at the beginning of each row. 


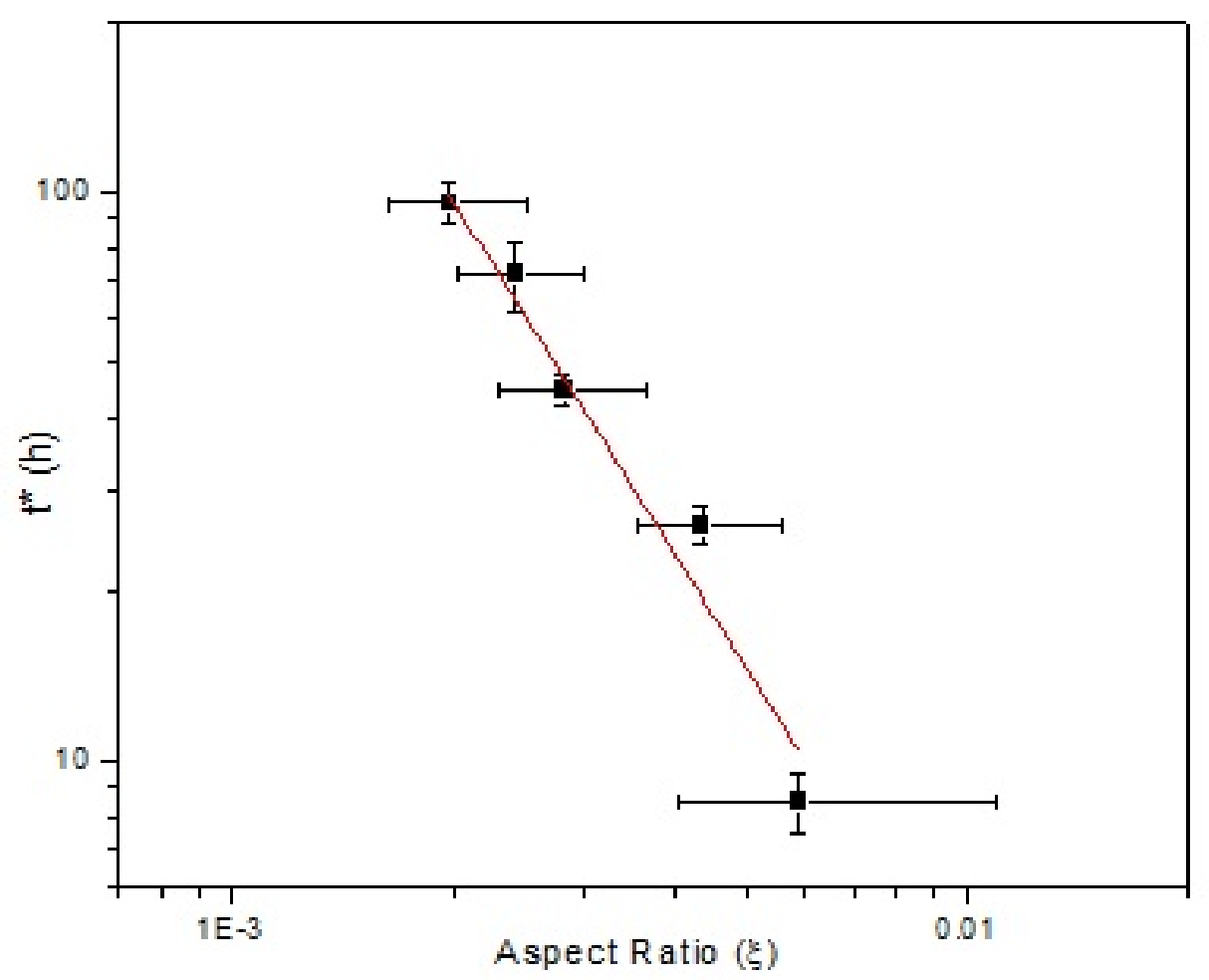

Figure 7. Isotropic-nematic (I-N) phase separation time ( $\mathrm{t}^{*}$, in hours) is plotted as a function of aspect ratio. The nematic fraction of the samples used to determine $t^{*}$ values was about $50 \%$.

the tactoids. If the sedimentation time for the tactoids is relatively short, nucleation time of the sample will determine the speed of the I-N phase separation. We argue that the nucleation rate of smaller size nanoplates is much faster and hence their I-N separation time is much less when compared to that of larger nanoplates. Nucleation of tactoids requires nanoplates to be oriented along the same direction from their initial disordered (metastable fluid) state to form a nematic tactoid. Therefore, tactoid nucleation is determined by the rotational and translational diffusion rate of nanoplates, which are inversely related to the platelet size. We approximate our $\mathrm{ZrP}$ nanoplates as oblate ellipsoid particles that follow the relations (Odriozola et al., 2004), $D_{\perp}^{R}=$ $\frac{3 k_{B} T}{2 \pi \eta d^{3}} \frac{\left(\xi^{-2}-2\right) S-1}{\xi^{-4}-1}, D_{\|}^{R}=\frac{3 k_{B} T}{2 \pi \eta d^{3}} \frac{\xi^{-2} S-1}{\xi^{-2}\left(\xi^{-2}-1\right)}$, where $D_{\perp}^{R}$ and $D_{\|}^{R}$ are rotational diffusion coefficients corresponding to small angular displacements around axes that are perpendicular and parallel, respectively, to the particle axial axis, and $D_{\|}^{T}=2 D_{\perp}^{T}=\frac{k_{B} T}{4 \pi \eta d} \frac{\left(3 \xi^{-2}-2\right) S-1}{\xi^{-2}-1}$, where $D_{\perp}^{T}$ and $D_{\|}^{T}$ are free translational diffusion coefficients parallel and perpendicular, respectively, to the particle axial axis; $k_{\mathrm{B}}$ is the Boltzmann coefficient, $\eta$ is the viscosity of the solvent, $\mathrm{T}$ is the absolute temperature, $d$ is the diameter of the platelets, $\xi$ is the aspect ratio defined as thickness/lateral size (diameter), and

$$
S=\left(\xi^{-2}-1\right)^{-1 / 2} \arctan \left(\sqrt{\xi^{-2}-1}\right) .
$$

This model, when applied to our highly anisotropic nanoplates $\left(\xi^{-1}\right.$ ranging from 170 to 400), simplifies to $D_{\perp}^{R}=D_{\|}^{R} \sim \frac{3 k_{B} T}{4 \eta d^{3}} \xi^{3}=\frac{3 k_{B} T}{4 \eta l^{3}} \xi^{6}$ and $D_{\|}=2 D_{\perp} \sim \frac{3 k_{B} T}{8 \eta d} \xi=\frac{3 k_{B} T}{8 \eta l} \xi^{2}$, where $l$ is the thickness of nanoplates. Clearly, nanoplates with smaller diameter (large $\xi$ ) have a much faster diffusion coefficient than nanoplates with larger diameter (small $\xi$ ), and hence tactoid nucleation takes place faster if the aspect ratio $(\xi)$ is large. Figure 6 supports this argument by demonstrating that the kinetics of transition from metastable fluid to I-N phase separation is the fastest in group 1 suspensions (largest $\xi$ ). 


\section{CONCLUSIONS}

In summary, due to faster rotational and translational diffusion rates, nanoplates with larger aspect ratio undergo faster nucleation of tactoids and hence form an I-N interface faster than smaller ones. I-N phase separation time $\left(\mathrm{t}^{*}\right)$ follows the relation, $t^{*}=\alpha\langle\xi\rangle^{n}$, where $\alpha=0.97 \pm 1.30 \mathrm{~s}$ and $n=-2.05 \pm 0.2$. Sedimentation speeds of tactoids of different aspect ratio can be studied in the future using microscopic observations of tactoids to further investigate the sedimentation process.

\section{ACKNOWLEDGEMENTS}

This work is supported by NASA under Grant NNX13AQ60G "Liquid Crystals of Nanoplates" and NSF under Grant No. DMR-1006870. Xuezhen Wang acknowledges support from Mary Kay O’Conner Process Safety Center, Texas A\&M University.

\section{REFERENCES}

Cheng Z, Chaikin PM, Zhu J, Russel WB, Meyer WV (2002) Crystallization kinetics of hard spheres in microgravity in the coexistence regime: interactions between growing crystallites. Physical Review Letters 88(1): 015501

Cheng Z, Russell WB, Chaikin PM (1999) Controlled growth of hard-sphere colloidal crystals. Nature 401: 893-895

Clearfield A, Stynes JA (1964) The preparation of crystalline zirconium phosphate and some observations on its ion exchange behavior. Journal of Inorganic \& Nuclear Chemistry 26(1): $117-129$

He P, Mejia AF, Cheng Z, Sun D, Sue HJ, Dinair DS, Marquez M (2010) Hindrance function for sedimentation and creaming of colloidal disks. Physical Review E: Statistical, Nonlinear, and Soft Matter Physics 81(2): 026310

Kim H-N, Keller SW, Mallouk TE, Schmitt J, Decher G (1997) Characterization of zirconium phosphate/ polycation thin films grown by sequential adsorption reactions. Chemistry of Materials 9(6): 1414-1421

Kleshchanok D, Holmqvist P, Meijer J-M, Lekkerkerker HNW (2012) Lyotropic smectic B phase formed in suspensions of charged colloidal platelets. Journal of the American Chemical Society 134(13): 59855990

Mejia AF, Chang Y-W, Ng R, Shuai M, Mannan MS, Cheng ZD (2012) Aspect ratio and polydispersity dependence of isotropicnematic transition in discotic suspensions. Physical Review E 85(6): 061708

Odriozola G, Romero-Bastida M, GuevaraRodriguez FJ (2004) Brownian dynamics simulations of laponite colloid suspensions. Physical Review E 70(2): 021405

Onsager L (1949) The effects of shape on the interaction of colloidal particles. Annals of the New York Academy of Sciences 51(4): 627-659

Shuai M, Mejia AF, Chang Y-W, Cheng Z (2013) Hydrothermal synthesis of layered alphazirconium phosphate disks: control of aspect ratio and polydispersity for nanoarchitecture. CrystEngComm 15(10): 19701977

Sun D, Sue H-J, Cheng Z, Martinez-Raton Y, Velasco E (2009) Stable smectic phase in suspensions of polydisperse colloidal platelets with identical thickness. Physical Review E 80(4): 041704

van der Kooij FM, Kassapidou K, Lekkerkerker HNW (2000) Liquid crystal phase transitions in suspensions of polydisperse plate-like particles. Nature 406: 868-871

Verhoeff AA, Bakelaar IA, Otten RHJ, van der Schoot P, Lekkerkerker HNW (2011) Tactoids of plate-like particles: size, shape, and director field. Langmuir 27(1): 116-125

Xu Z, Gao C (2011) Graphene chiral liquid crystals and macroscopic assembled fibres. Nature Communications 2: 571

Zhu J, Li M, Rogers R, Meyer W, Ottewill RH, Space Shuttle Crew STS-73, Russell WB, Chaikin PM (1997) Crystallization of hardsphere colloids in microgravity. Nature 387: 883-885 